\title{
CD34 enrichment of umbilical cord blood
}

\author{
Pafumi C \\ Department of Obstetrical Science University of Catania, Italy.pafumi@unict.it
}

\begin{abstract}
Objective: We describe the relation between the clamping time and blood volume collected, plus two enrichment systems for CD34+ stem cells from umbilical cord blood, to achieve an excellent recovery of cells with a high proliferative ability and bone marrow reconstitution.

Method: After cord blood collection by an obstetrician, stem cell purification has been performed either with a combination of monoclonal antibodies, using the negative selection Stem Sep method, or with a positive cell selection based on their surface CD34 antigens, using the Mini Macs system.

Results: An excellent recovery of hematopoietic progenitors: Burst Forming Unit Erythroid; Colony Forming Unit Granulocyte and Macrophage; and Colony Forming Unit Granulocyte, Erythroid, Monocyte and Macrophage, inversely related to the rising of clamping time, has been achieved with the Mini Macs system (54\% of colonies, with $90 \%$ purity). With the Stem Sep method, recovery of hematopoietic progenitors was $35 \%$ (with $80 \%$ purity). Conclusions: By an early clamping of umbilical cord blood, we obtained a higher number of CD34+ cells whose clonogenic activity increased with enrichment. This is particularly useful considering that the number of CD34+ stem cells contained in a unit of placental blood is enough for transplantation to a child, but not for an adult engraftment. Thus, using these methods, we can obtain a larger number of CD34+ stem cells, which increases the possibility of reducing graft-versus-host disease in adult patients, producing survival rates similar to those of transplantation of bone marrow from unrelated donors (Ref. 5). Full Text in PDF www.elis.sk.

Key words: umbilical cord blood, transplantation of bone marrow from unrelated donors, graft-versus-host disease.
\end{abstract}

Précis: An enrichment system for CD34+ stem cell collection from umbilical cord blood enhances the number of transplantable cells, increasing the possibility of adult engraftment.

Hematopoietic cells are heterogeneous, with different lineage at different stages of maturity. The structural and functional integrity of hematopoietic system is maintained by CD34+ stem cells, which can self-renew, produce other stem cells or differentiate to produce various hematopoietic lineages (1). Hematopoietic progenitor stem cell transplantation is an important therapy for certain haematological and malignant disorders. Both umbilical cord blood and placental blood contain a high number of hematopoietic progenitor cells, which possess significant clinical advantages over bone marrow, in terms of proliferative capacity and immunologic reactivity (2). This source of hematopoietic stem cells is characterized by a relative ease of procurement, absence of risk to the donor, and a small likelihood of transmitting cytomegalovirus and Epstein Barr virus infections (3). Umbilical cord blood can be given in vivo to fully and partially HLA-matched sibling or nonfamilial recipients for bone marrow reconstitution in genetic disorders as

Department of Obstetrical Science University of Catania, Italy

Address for correspondence: C. Pafumi, MD, Institute of Obstetrics and Gynaecology, University of Catania, Via G. D’Annunzio No. 125, IT-951 27 Catania, Italy.

Acknowledgement: Valentina Pafumi has carried out English language editing for this article. well as malignancies. In comparison to adult peripheral blood, umbilical cord blood displays decreased immune responses to alloantigens. Although low incidence of graft-versus-host disease occurs, a sufficient graft-versus-leukemia activity remains, which is important for the risk of relapse and mortality (4). However, a unit of placental blood, collected from a single donor, contains a relatively low number of CD34+ cells after processing and testing. There may be enough hematopoietic stem cells for use in children, but the ability to engraft an adult might require ex vivo manipulation (5). The aim of this study was to improve the feasibility of an obstetrician-based cord blood collection system and an efficient CD34+ cells recovery. We have developed a technique which flushes and collects placental derived stem cells, using two systems of CD34+ cell purification: a negative selection with the Stem Sep method and a positive selection with Mini Macs. In this study, an earlier blood collection permits the collection of a larger number of hematopoietic progenitor cells, avoiding an early stasis phenomena and intravascular coagulation, and reducing the transfer of red blood cells. The clamping time was calculated from the foetus birth till the clamping of umbilical cord blood with clips. The umbilical cord blood volume $(120 \mathrm{ml})$ increased with a plateau between $20-30 \mathrm{sec}$.

\section{Materials and methods}

The Institute of Obstetrics and Gynecology, University of Catania, S. Bambino Hospital obtained, after an informed consent and 
according to institutional guidelines, umbilical cord blood from 47 patients. Placental blood units were collected from freshly delivered placentas in preservative-free ethylenediaminetetraacetic acid anticoagulant (EDTA). Data were obtained from the mothers' interviews and the mothers' and infants' medical records. For the purposes of consent and analysis, the mother was considered to be the donor. The umbilical cord blood, in preservative-free EDTA anticoagulants, was collected after the delivery of an infant and the ligation of the cord, before placental detachment. After the blood flow from the cord, with the baby kept under the level of his mother's perineal region, the cord was doubly clamped by means of a hemostatic clamp a few centimetres from the umbilicus. The distal portion of the umbilical cord was cleaned with iodate alcohol (Betadine solution and alcohol), the umbilical vein was cannulated, and the sample collected by gravity into a sterile set for placental collection. A higher quantity was collected when the sampling was performed before placental detachment, both in vaginal deliveries and in caesarean sections. Residual maternoplacental circulation represented a "Vis a tergo" that permitted blood to flow from the maternal section of placenta: in addition, there were uterine contractions during placental stage. The blood quantity collected depended on:

- The period of gestation: the volume of umbilical cord blood collected, which depends on placental weight and foetal weight, is greater with longer gestation;

- Placental infarction: decreased placental functional surface yields a minimal quantity of blood;

- Foetal respiratory distress: during foetal hypoxia in labour, a greater quantity of blood flows from placenta to foetus, with less placental blood remaining after delivery;

- Clamping time: if the cord is clamped sooner and before placental separation, less blood is transferred to the infant and thus more is available for cord blood collection.

The earlier blood collection permitted a greater quantity of blood collected, avoiding the early stasis phenomena and intravascular coagulation. Once collected, the blood was separated over a Ficoll-Hypaque density gradient (d-1.077) at $400 \mathrm{~g}$ for 40 minutes at $20^{\circ} \mathrm{C}$ : interface mononuclear cells were collected, twice washed, and resuspended in the Iscove's modified Dulbecco's medium. Cell vitality was assessed by the Trypan blue exclusion test; cell mortality never exceeded $10 \%$. Cord blood mononuclear cells were resuspended in the Iscove's modified Dulbecco's medium containing $20 \%$ heat-inactivated foetal calf serum at a cellular density of $2 \times 10^{6}$ cells $/ \mathrm{ml}$ and then treated with overnight plastic adherence cycles. Cells were washed three times, resuspended in PBS and prepared for Mini Macs or Stem Sep separation. CD34+ cells then were resuspended in a small volume of medium, counted, and plated in semisolid medium for clonogenic assays and a liquid culture as indicated below. Purification of CD34+ cells from total cord blood was obtained either with a combination of monoclonal antibodies, glycoforina A, CD3, CD2, CD56, CD24, CD19, CD14, CD16, CD66, using the Stem Sep negative selection method, or positive selection of CD34 expressing cells using a Mini Macs system. An excellent recovery of hematopoietic progenitors (with a CD34+ purity of $99 \%$ ) and hematopoietic colony forming cells (Burst Forming Unit Erythroid; and Colony Forming Unit Granulocyte and Macrophage) were obtained with the Mini Macs system. Cells purified with either the Mini Macs or Stem Sep system, were plated in methylcellulose culture at a density of $1 \times 10^{3}$ cells for plates (two plates per point), in $0.9 \%$ methylcellulose, and $3.0 \mathrm{UI} /$ $\mathrm{ml}$ erythropoietin, granulocyte and macrophage stem cell factor $(10 \mathrm{ng} / \mathrm{ml})$, and interleukin $3(100 \mathrm{U} / \mathrm{ml})$ in the Iscove's modified Dulbecco's medium supplemented with A-thioglycerolo $\left(10^{-4} \mathrm{~mol} /\right.$ $\mathrm{ml}$ ) at $37{ }^{\circ} \mathrm{C}$ in a $5 \% \mathrm{CO}_{2} / \mathrm{O}_{2}$ humidified atmosphere. Colonies were categorized after 14 days.

\section{Results}

The role of the clamping time in modifications of umbilical cord blood was studied. The clamping time was calculated from the foetus birth till the clamping of umbilical cord blood with clips. The umbilical cord blood volume collected increased when clamping was effected sooner, with a "plateau" between 20 and 30 seconds. The volume of detected umbilical cord blood was equal when the clamping time increased over 30 seconds. White blood cells, red blood cells, and haematocrit of newborn increased as clamping time increased. A greater blood quantity was obtained when collection was performed before placental detachment, both in vaginal deliveries and in caesarean sections under ultrasonic guidance. Residual materno-placental circulation represented a "Vis a tergo" that permitted blood to flow from the foetal sections of placenta to umbilical cord; in addition there were uterine contractions during placental stage. The early blood collection permitted a larger blood quantity collection, anticipating the early stasis phenomena and intravascular coagulation.

We studied the effect of the clamping time on the number of $\mathrm{CD} 34+$ cells isolated. Our results are representative of 50 experiments. Hematopoietic progenitors were purified from total cord blood using two methods: Mini Macs separation (the final index of purification was $99 \%$ ), and Stem Sep method (obtaining a purity of $80 \%$ ).

The effects of clamping time on clonogenic capacity were related to the timing of blood collection. The purified umbilical cord blood progenitors included a majority of Burst Forming Unit Erythroid and Colony Forming Unit Granulocyte and Macrophage colony and a minority of multipotent progenitors Colony Forming Unit Granulocyte, Erythroid, Monocyte and Macrophage. These progenitors gave rise to large colonies in clonogenic culture. The number of colonies was hgher with Mini Macs system (54 \%) than with the negative selection Stem Sep system (35\%). In both cases, each colony contained 105 cells.

\section{Discussion}

Hemopoietic stem cells are increasingly used for the treatment of malignant and non-malignant disorders. Various attempts have been made in recent years to expand and manipulate these cells in order to increase their therapeutic potential. Cord blood is a particularly attractive alternative to bone marrow or to peripheral 
$386-388$

blood cells mobilized by growth factors as a source of transplantable hematopoietic tissue. This study was aimed to improve the feasibility of an obstetrician-based cord blood collection system and the enrichment of CD34+ progenitor cord blood to obtain an excellent hematopoietic progenitor recovery. It has been reported that an early collection would permit a larger quantity to collect (anticipating an early stasis phenomena and intravascular coagulation). This method has several clinical advantages: a) if a newborn is underweight (preterm), the umbilical cord must be cut immediately to avoid risks related to an abrupt circulatory overload and to permit reanimation as soon as possible; b) immediate umbilical cord clamping is needed in red cell materno-fetal isoimmunization to reduce the transfer of foetal blood containing red cells with superficial antigens to his mother; $\mathrm{c}$ ) if the mother is RH negative, it should be helpful not to occlude the umbilical cord near the placenta after the cut and to reduce the possible passage of foetal red cells to maternal circulation. With this study, which has been performed on 50 cord blood units, we determined that an efficient recovery of CD 34+ cells from UCB was obtained by the early clamping. The blood volume collected was related to the increase of clamping time, with the "plateau" between 20 and 30 seconds. Using the magnetic cell sorting Mini Macs or Stem Sep system to isolate CD34+ cells and demonstrating an excellent recovery of hematopoietic progenitors and hematopoietic colony forming cells when the umbilical cord blood cut was made precociously, we studied the enrichment of hematopoietic progenitors.

\section{Conclusions}

By applying the early clamping of umbilical cord blood, we obtained a higher number of CD34+ cells. Their clonogenic activity increased with enrichment. By using a positive selection (Mini Macs method) we got better results (54\% of colonies) than by using a negative selection (Stem Sep system: $35 \%$ of colonies), getting a purity of $99 \%$ instead of $80 \%$. This is particularly useful considering that the number of CD34+ stem cells contained in one unit of placental blood is enough for transplanting to a child, but not for an adult engraftment. Thus, by using these methods, we can isolate a higher number of stem cells, which increase the possibility of engraftment in adult patients, producing survival rates similar to those obtained with transplantation of bone marrow from unrelated donors. Thus, umbilical cord blood may be considered a useful source of hematopoietic stem cells for patients who do not have an HLA related donor.

\section{References}

1. Morrison SJ, Shah NM, Anderson DJ. Regulatory mechanism in stem cell biology. Clee 1997; 88: 287-298.

2. Rubinstein P, Carrier C, Scaradavou A, Kurtzberg J, Adamson J, Migliaccio AR et al. Outcomes among 562 recipients of placental blood transplants from unrelated donors. N Engl J Med 1998; 339: 1565-1577.

3. Almici C, Carlo-Stella, Wagner JE, Mangoni L, Garau D, Rizzoli V. Biologic and phenotypic analysis of early haematopoietic progenitor cells in umbilical cord blood. Leukemia 1997: 11 (12): 21-43.

4. David S, Boiron JM, Rice A, Vianes I, Duperray V, Reiffers J. Expansion of blood CD34+ cells: committed precursor expansion does not affect immature haematopoietic progenitors. J Hematother 1997; 6 (2): 151-158.

5. Farley TJ, Ahmed T, Fitzgerald M, Preti RA. Optimisation of CD34+ cell selection using immunomagnetic beads: implications for use in cryopreserved peripheral blood stem cell collection. J Hematother 1997; 6 (1): 53-60.

Received September 19, 2011. Accepted November 30, 2011. 